\title{
Funcionalidade e força muscular estão associadas ao risco e medo de quedas em idosos?
}

\author{
Are functionality and muscle strength associated with risk and fear of falls in the \\ elderly?
}

\section{¿Están la funcionalidad y la fuerza muscular asociadas con el riesgo y el miedo de caídas en mayores?}

\author{
Daniel Vicentini de Oliveira (iD \\ Universidade Estadual de Maringá - Maringá (PR) - Brasil
}

\author{
Naelly Renata Saraiva Pivetta iD \\ Universidade Estadual de Campinas - Campinas (SP) - Brasil
}

Felipe Carmona Yamashita iD

Centro Universitário Metropolitano de Maringá - Maringá (PR) - Brasil

Matheus Amarante do Nascimento (iD

Universidade Estadual do Paraná - Paranavaí (PR) - Brasil

Natália Quevedo dos Santos (iD

Universidade Cesumar - Maringá (PR) - Brasil

José Roberto Andrade do Nascimento Júnior (iD

Universidade Federal do Vale do São Francisco - Petrolina (PE) - Brasil

Sônia Maria Marques Gomes Bertolini iD

Universidade Cesumar - Maringá (PR) - Brasil

\section{RESUMO}

Objetivo: Investigar se o nível de funcionalidade e a força muscular estão associados ao risco de queda e ao medo de cair em idosos. Métodos: Estudo transversal, realizado com 80 idosos, de março a agosto de 2019, no município de Maringá, Paraná, Brasil. Para avaliação, aplicaram-se: um questionário sociodemográfico, o WHODAS 2.0, a Escala Internacional de Eficácia de Quedas, o teste de levantar e sentar na cadeira, o teste de flexão de antebraço, além do teste sentado, caminhada de $2,44 \mathrm{~m}$ e voltar a sentar. Para análise dos dados, utilizaram-se a correlação de Pearson e análise de regressão múltipla $(p<0,05)$. Resultados: Os domínios de funcionalidade e a força muscular explicam $40 \%$ da variância nas pontuações do medo de quedas, no entanto apenas os domínios de autocuidado $(\beta=0,409)$ e AVD $(\beta=0,379)$ apresentaram associação significante $(p<0,05)$ e positiva com o medo de quedas. Os domínios de funcionalidade e a força muscular explicam $51 \%$ da variância das pontuações do risco de queda, sendo que apenas o domínio de relações interpessoais $(\beta=-0,340)$, a força muscular de membros superiores $(\beta=-0,512)$ e a força muscular de membros inferiores $(\beta=-0,192)$ apresentaram associação significante $(p<0,05)$ e negativa com o risco de quedas. Conclusão: Observa-se que, na população de idosos em questão, as medidas de funcionalidade e a força muscular estão associadas ao risco de queda e ao medo de cair.

Descritores: Idoso; Exercício Físico; Equilíbrio postural; Classificação Internacional de Funcionalidade, Incapacidade e Saúde.

\section{ABSTRACT}

Objective: To investigate whether the level of functionality and muscle strength is associated with the risk of falling and the fear of falling in the elderly. Methods: Cross-sectional study, carried out with 80 elderly, from March to August 2019, in the municipality of Maringá, Paraná, Brazil. For evaluation, the following were applied: a sociodemographic questionnaire, the WHODAS 2.0, the International Scale for the Effectiveness of Falls, the test of getting up and sitting on the chair, the forearm flexion test, in addition to the sitting test, walking of $2.44 \mathrm{~m}$ and sit back. For data analysis, we used Pearson's correlation and multiple regression analysis $(p<0.05)$. Results: The domains of functionality and muscle strength explain $40 \%$ of the variance of the fear of falling scores; however,

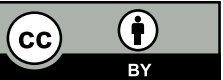


only the domains of self-care $(\beta=0.409)$ and $A D L(\beta=0.379)$ showed a significant $(p<0.05)$ and positive association with the fear of falls. The domains of functionality and muscle strength explain $51 \%$ of the variance of the risk scores for falling, with only the domain of interpersonal relationships $(\beta=-0.340)$, the muscular strength of upper limbs $(\beta=-0.512)$, and strength lower limb muscle $(\beta=-0.192)$ showed a significant $(p<0.05)$ and negative association with the risk of falls. Conclusion: It is observed that in the elderly population in question, measures of functionality and muscle strength are associated with the risk of falling and the fear of falls.

Descriptors: Elderly; Exercise; Postural Balance; International Classification of Functioning, Disability and Health.

\section{RESUMEN}

Objetivo: Investigar si el nivel de la funcionalidad y la fuerza muscular están asociados con el riesgo de caída y el miedo de los mayores en caerse. Métodos: Estudio transversal realizado con 80 mayores entre marzo y agosto de 2019 en el municipio de Maringá, Paraná, Brasil. Se ha aplicado para la evaluación los instrumentos a continuación: un cuestionario sociodemográfico, el WHODAS 2.0, la Escala Internacional de Eficacia de Caídas, la prueba de sentarse y levantarse de la silla, la prueba de flexión de antebrazo además de la prueba sentada, la caminata de 2,44m y volver a sentarse. Se ha utilizado la correlación de Pearson y el análisis de regresión múltiple $(p<0,05)$ para el análisis de datos. Resultados: Los dominios de la funcionalidad y la fuerza muscular explican el $40 \%$ de la variancia de las puntuaciones del miedo de caídas, sin embargo, solamente los dominios de autocuidado $(\beta=0,409)$ y AVD $(\beta=0,379)$ presentaron asociación significante $(p<0,05)$ y positiva con el miedo de caídas. Los dominios de funcionalidad y la fuerza muscular explican el $51 \%$ de la variancia de las puntuaciones del riesgo de caída y solamente los dominios de las relaciones interpersonales $(\beta=-0,340)$, la fuerza muscular de los miembros superiores $(\beta=-0,512)$ y la fuerza muscular de los miembros inferiores $(\beta=-0,192)$ presentaron asociación significante $(p<0,05)$ y negativa con el riesgo de caídas. Conclusión: Se observa que en esa población de mayores las medidas de funcionalidad y de fuerza muscular se asocian con el riesgo de caída y el miedo de caerse.

Descriptores: Anciano; Ejercicio Físico; Equilibrio postural; Clasificación Internacional del Funcionamiento, de la Discapacidad y de la Salud.

\section{INTRODUÇÃO}

O envelhecimento provoca alterações no organismo humano, afetando especialmente a força muscular, a qual tende a reduzir, contribuindo para a diminuição do equilíbrio e da independência funcional ${ }^{(1)}$. A força pode ser compreendida como a habilidade do músculo em vencer uma resistência. Já o equilíbrio é um componente de aptidão física relacionado com a habilidade de manter a estabilidade estática e dinâmica( ${ }^{(2)}$. A independência funcional, por sua vez, está relacionada à capacidade física de realizar atividades funcionais, importantes para manutenção da vida cotidiana, sem depender parcial ou totalmente de auxílio de terceiros ${ }^{(3)}$.

Essas variáveis são componentes importantes da promoção de saúde, pois, quando preservadas, possibilitam que o idoso realize seu autocuidado e suas atividades cotidianas de forma mais segura e eficaz. Quando prejudicadas, elas podem desencadear quedas, as quais têm sido foco de diversas investigações nos últimos anos ${ }^{(4-8)}$.

A queda, atualmente, é considerada um problema grave de saúde pública e, de acordo com dados da Organização Mundial da Saúde, aproximadamente $30 \%$ dos idosos sofrem quedas em um período de um ano, o que, por sua vez, pode levar ao aumento de lesões, distúrbios emocionais e óbito( ${ }^{(9)}$.

A queda está relacionada a circunstâncias multifatoriais, intrínsecas ou extrínsecas. Os fatores intrínsecos são caracterizados por alterações fisiológicas inerentes ao envelhecimento, distúrbios sensoriais e cognitivos, disfunções neuromusculares e doenças, que afetam o equilíbrio e a marcha ${ }^{(10)}$. Entre os fatores intrínsecos, podem-se destacar: a idade avançada propriamente dita, comorbidades, doenças osteomioarticulares, depressão, baixa autoeficácia para evitar quedas ${ }^{(11)}$ e baixa acuidade visual(6). Os fatores extrínsecos, por sua vez, estão relacionados aos riscos oferecidos pelo ambiente, tais como: superfícies irregulares, piso escorregadio, iluminação inadequada, escada sem corrimão( ${ }^{(12)}$, tapete desprendido do chão e degrau elevado ou estreito ${ }^{(6)}$.

Os fatores extrínsecos estão frequentemente relacionados à ocorrência de uma única queda, uma vez que são modificáveis ${ }^{(12)}$. Já os fatores intrínsecos não são passíveis de mudança ou de cura (no caso de doenças crônicas, por exemplo), por isso podem resultar em fraturas, entorses e lesões, levando o idoso a apresentar medo de cair( ${ }^{(13)}$, o qual pode ser definido como uma preocupação em se ter uma queda, levando o idoso a evitar muitas atividades no seu dia a dia ${ }^{(14)}$.

Já é bem estabelecido na literatura que as quedas têm um impacto significativo em alguns aspectos da vida do idoso, acarretando prejuízos na qualidade de vida e custos elevados ${ }^{(5-8)}$. Estima-se que aproximadamente $70 \%$ dos 
idosos que sofreram uma queda desenvolveram medo de cair, e que, quanto maior a idade, maior a prevalência do medo de cair, havendo predomínio no sexo feminino(10). Ter medo de cair afeta a autonomia, a independência, 0 desempenho das atividades diárias e facilmente expõe o idoso ao isolamento social, insegurança e diminuição na funcionalidade e, consequentemente, da força muscular ${ }^{(15)}$.

A literatura propõe que o medo de cair leva o idoso a reduzir a frequência e intensidade de suas atividades cotidianas, contribuindo para a redução da funcionalidade e da força muscular; no entanto o contrário também é verdadeiro, evidenciando que idosos fisicamente inativos apresentam déficit de força e funcionalidade em relação a idosos fisicamente ativos. Essas duas variáveis, "força" e "funcionalidade", são extremamente importantes em se tratando de idosos, pois são capazes de fornecer indícios do estado de saúde e qualidade de vida do idoso(6).

Explorar o nível de funcionalidade e força muscular e a sua relação com uma potencial queda e o medo de cair pode ajudar na identificação de perfis de idosos caidores e com medo de cair e, a partir disso, traçar um plano de avaliação e intervenções específicas. Diante do exposto, este estudo tem por objetivo investigar se o nível de funcionalidade e a força muscular estão associados ao risco de queda e ao medo de cair em idosos, uma vez que os resultados obtidos subsidiarão medidas profiláticas e intervencionistas, a fim de promover a saúde de idosos.

\section{MÉTODOS}

Trata-se de uma pesquisa observacional, transversal e analítica, que seguiu os procedimentos, etapas e diretrizes do STROBE Statement. Realizou-se a coleta de dados de março a agosto de 2019, em cinco academias da terceira idade (ATI) espalhadas pelo município de Maringá, Paraná, Brasil, em horários diversos (manhã, tarde e/ou noite), de acordo com a disponibilidade do pesquisador. Do total de ATI do munícipio, sortearam-se cinco localizadas por regiões específicas (norte, sul, leste, oeste e centro da cidade).

Ao aproximar-se do idoso, o pesquisador explicou os procedimentos e objetivos da pesquisa. Os idosos que aceitaram participar assinaram um Termo de Consentimento Livre e Esclarecido (TCLE).

A amostra, escolhida de forma intencional e por conveniência, compôs-se de 80 idosos de ambos os sexos. Incluíram-se idosos frequentadores das academias da terceira idade (ATI) do município de Maringá, Paraná, não praticantes de exercício físico (as ATI do município não possuem profissionais de educação física em período integral, para poder padronizar e prescrever exercícios para os idosos, diante disso, elas se enquadram como locais de atividade física, e não de exercício físico).

Tiveram que ser excluídos os idosos com possíveis déficits cognitivos, avaliados por meio do Mini Exame do Estado Mental (MEEM). As notas de corte para exclusão pelo MEEM foram: abaixo de 17 para os analfabetos; 22 para idosos com escolaridade entre 1 e 4 anos; 24 para os com escolaridade entre 5 e 8 anos, e 26 para os que tiverem 9 anos ou mais anos de escolaridade ${ }^{(16)}$. Também se excluíram os idosos com possíveis disfunções que impedissem a colaboração na realização do estudo (compreensão e resposta, por exemplo) autopercebidas pelos avaliadores. Excluíram-se apenas dois idosos.

Realizou-se a coleta de dados na forma de entrevista pelos pesquisadores, ou seja, os questionários eram lidos para os idosos, evitando erros de interpretação e leitura pelos pesquisados. A entrevista durou, em média, 15 minutos por idoso.

Para avaliar o perfil sociodemográfico e de saúde dos idosos, utilizou-se um questionário elaborado pelos próprios autores, com questões relacionadas com: a idade, a faixa etária (60 a 70 anos; 71 a 80 anos; 81 a 90 anos; mais de 90 anos), o sexo (masculino; feminino), o estado civil (com companheiro/a; sem companheiro/a), a renda mensal em salários mínimos (SM) (1 a 2 SM; 2,1 a 3 SM; mais de 3 SM), a aposentadoria (sim; não), a percepção da saúde atual (boa; regular; ruim), o histórico de queda nos últimos seis meses (sim; não - essa questão foi feita perguntando se o idoso caiu no chão nos últimos seis meses) e quanto tempo pratica atividades na ATI (menos de 3 meses; 3 meses a 1 ano; 1 a 5 anos; mais de 5 anos).

Avaliou-se o medo de cair pela Escala Internacional de Eficácia de Quedas (FES-I). Esse questionário contém 16 domínios, com diferentes AVD, com quatro possibilidades de resposta e respectivos escores de um a quatro ("nem um pouco preocupado" a "extremamente preocupado"). O escore total pode variar de 16 a 64, da ausência de preocupação até a preocupação extrema, respectivamente, em relação às quedas durante a realização das atividades especificadas no questionário( ${ }^{(17)}$.

Avaliou-se a funcionalidade por meio da Avaliação de Saúde e Deficiência WHODAS 2.0. Esse instrumento avalia a funcionalidade em seis domínios de atividade: cognição, mobilidade, autocuidado, relações interpessoais, 
atividades diárias e participação, a partir de 12 questões. Cada item avalia a quantidade de dificuldade que o idoso apresenta, no período do último mês, para realizar suas atividades. Cada questão possui uma escala Likert de pontos de 0 (nenhuma dificuldade) a 4 (extrema dificuldade). Ao somar as duas questões de cada domínio, tem-se um escore final, que pode variar de 0 a 8 , sendo que, quanto maior a soma, maior a dificuldade (incapacidade) para realização do domínio avaliado(18).

Avaliou-se, ainda, a força muscular de membro superior pelo teste de flexão de antebraço e a força muscular de membro inferior pelo teste de levantar e sentar na cadeira. Realizou-se o teste sentado, caminhada de $2,44 \mathrm{~m} e$ voltar a sentar para avaliar a mobilidade física - velocidade, agilidade e equilíbrio dinâmico ${ }^{(19)}$.

Realizou-se a análise descritiva por meio das medidas de tendência central (média) e dispersão (mínimo, máximo, desvio padrão, assimetria e curtose) para descrever as variáveis do estudo. Utilizou-se a correlação de Pearson para investigar a relação entre a funcionalidade, a força muscular, o risco de queda e o medo de queda; além da análise de regressão múltipla para determinar se a funcionalidade e a força muscular predizem o medo e o risco de quedas. Conduziram-se dois modelos para investigar a predição dos domínios de funcionalidade e a força muscular (variáveis independentes) sobre as pontuações do risco de queda (Modelo 1) e medo de queda (Modelo 2). Todas as variáveis independentes foram incluídas juntas no modelo no mesmo bloco e os dados rastreados para garantir que as suposições de matrizes de normalidade, linearidade, multicolinearidade e homogeneidade de variância-covariância fossem atendidas ${ }^{(20)}$. Não houve correlações suficientemente fortes entre as variáveis que indicassem problemas de multicolinearidade (fatores de inflação de variância $<5,0$ ). Utilizou-se o software SPSS, versão 22.0, em todas as análises.

Esta pesquisa obteve a aprovação do comitê de ética e pesquisa do Centro Universitário Metropolitano de Maringá por meio do Parecer n. ${ }^{\circ} 3.632 .033 / 2019$.

\section{RESULTADOS}

Dos 80 idosos avaliados, nota-se (Tabela I) a prevalência de idosos do sexo feminino $(63,7 \%)$, com companheiro $(69,2 \%)$, com idade entre 60 e 70 anos $(62,5 \%)$, renda mensal a partir de dois salários mínimos $(62,5 \%)$ e que são aposentados $(65,0 \%)$. Nota-se que a maioria dos idosos possui percepção de saúde regular/ruim $(56,3 \%)$, não tem histórico de quedas $(63,7 \%)$, frequenta as ATI há mais de 1 ano $(73,3 \%)$ e com frequência de 2 vezes ou mais por semana $(76,0 \%)$.

Os resultados apresentados na Tabela II demonstram os valores descritivos dos domínios de funcionalidade, a força muscular de membros superiores e inferiores, o medo de cair e o risco de queda dos idosos.

De acordo com a Tabela III, verificaram-se as seguintes correlações significativas $(p<0,05)$ : do medo de queda com a cognição $(r=0,56)$, a mobilidade $(r=0,50)$, o autocuidado $(r=0,58)$, a AVD $(r=0,59)$, a participação social $(r=0,51)$; com o escore total de funcionalidade $(r=0,60)$ demonstrou uma correlação moderada; já com as relações interpessoais $(r=0,42)$ demonstrou uma correlação fraca.

Em relação ao risco de queda, correlacionou-se significativamente $(p<0,05)$ e fraco com a mobilidade $(r=0,40)$, AVD ( $r=0,33)$, a participação social $(r=0,35)$, o escore total de funcionalidade $(r=0,31)$; e de modo moderado com a força muscular de membros superiores $(r=-0,59)$ e de membros inferiores $(r=-0,62)$.

Os resultados obtidos (Tabela IV) indicam que os domínios de funcionalidade e as variáveis de força muscular explicam $40 \%$ da variância das pontuações do medo de queda, no entanto apenas os domínios de autocuidado $(\beta=0,409)$ e AVD $(\beta=0,379)$ apresentaram associação significante $(p<0,05)$ e positiva com o medo de queda. Destaca-se que a piora da funcionalidade nos referidos domínios está associada com a piora no medo de cair dos idosos. (Tabela IV).

Os resultados apontados na Tabela $V$ indicam que os domínios de funcionalidade e as variáveis de força muscular explicam $51 \%$ da variância das pontuações do risco de queda, no entanto apenas o domínio de relações interpessoais $(\beta=-0,340)$ e a força muscular de membros superiores $(\beta=-0,512)$ e de membros inferiores $(\beta=-0,192)$ apresentaram associação significante $(p<0,05)$ e negativa com o risco de queda. Destaca-se que, quanto maior a força muscular, principalmente de membros superiores, menor o risco de queda dos idosos. Nota-se, também, uma associação inversamente proporcional do domínio de funcionalidade de relações interpessoais com o risco de queda, isto é, o aumento no escore desse domínio (pior funcionalidade) leva à redução do escore do risco de queda (menor o risco). 
Tabela I - Perfil dos idosos usuários das ATI do município de Maringá, Paraná, Brasil, 2019.

\begin{tabular}{|c|c|c|}
\hline Variáveis & $\mathbf{n}$ & $\%$ \\
\hline \multicolumn{3}{|l|}{ Sexo } \\
\hline Masculino & 29 & 36,3 \\
\hline Feminino & 51 & 63,7 \\
\hline \multicolumn{3}{|l|}{ Estado civil ${ }^{a}$} \\
\hline Com companheiro & 54 & 69,2 \\
\hline Sem companheiro & 24 & 30,8 \\
\hline \multicolumn{3}{|l|}{ Faixa etária } \\
\hline 60 a 70 anos & 50 & 62,5 \\
\hline Mais de 70 anos & 30 & 37,5 \\
\hline \multicolumn{3}{|l|}{ Renda mensal ${ }^{a}$} \\
\hline 1 a 2 SM & 18 & 37,5 \\
\hline 2,1 a $3 \mathrm{SM}$ & 11 & 22,9 \\
\hline Mais de 3 SM & 19 & 39,6 \\
\hline \multicolumn{3}{|l|}{ Aposentadoria ${ }^{a}$} \\
\hline Sim & 52 & 65,0 \\
\hline Não & 25 & 35,0 \\
\hline \multicolumn{3}{|l|}{ Percepção de saúde } \\
\hline Boa & 35 & 43,8 \\
\hline Regular/ruim & 45 & 56,3 \\
\hline \multicolumn{3}{|c|}{ Quedas nos últimos 6 meses } \\
\hline $\operatorname{Sim}$ & 29 & 36,3 \\
\hline Não & 51 & 63,7 \\
\hline \multicolumn{3}{|c|}{ Frequência semanal nas $A T I^{a}$} \\
\hline 1 a 2 vezes & 18 & 24,0 \\
\hline 2 a 3 vezes & 37 & 49,3 \\
\hline Mais de 3 vezes & 20 & 26,7 \\
\hline \multicolumn{3}{|l|}{ Tempo de prática ${ }^{a}$} \\
\hline Até 1 ano & 20 & 26,7 \\
\hline 1,1 a 5 anos & 30 & 40,0 \\
\hline Mais de 5 anos & 25 & 33,3 \\
\hline
\end{tabular}

a: Variáveis com casos ausentes; ATI: academias da terceira idade; SM: salário mínimo

Tabela II - Análise das variáveis da pesquisa. Maringá, Brasil, 2019.

\begin{tabular}{lccc}
\hline Variáveis & Mínimo & Máximo & x (dp) \\
\hline Funcionalidade & & & \\
Cognição & 2,00 & 8,00 & $2,84(1,24)$ \\
Mobilidade & 2,00 & 8,00 & $3,15(1,49)$ \\
Autocuidado & 2,00 & 6,00 & $2,45(0,87)$ \\
Relações interpessoais & 2,00 & 7,00 & $2,53(0,95)$ \\
Atividades da vida diária & 2,00 & 8,00 & $2,83(1,30)$ \\
Participação social & 2,00 & 6,00 & $2,70(1,05)$ \\
Escore total & 12,00 & 41,00 & $16,49(5,99)$ \\
Força muscular MMSS (repetiçöes) $_{\text {Força muscular MMII (repeticöes) }}$ & 18,00 & 27,00 & $23,07(1,88)$ \\
Medo de cair & 10,00 & 20,00 & $15,91(2,11)$ \\
Risco de queda & 16,00 & 56,00 & $25,55(8,14)$ \\
\hline
\end{tabular}

X: média; dp: desvio-padrão; MMSS: membros superiores; MMII: membros inferiores 
Tabela III - Correlação entre a funcionalidade, a força muscular, o medo de queda e o risco de queda dos idosos. Maringá, Paraná, Brasil, 2019.

\begin{tabular}{|c|c|c|c|c|c|c|c|c|c|c|c|}
\hline \multirow[t]{2}{*}{ Variáveis } & \multicolumn{7}{|c|}{ Funcionalidade } & \multicolumn{2}{|c|}{$\begin{array}{c}\text { Força } \\
\text { muscular }\end{array}$} & \multicolumn{2}{|c|}{ Quedas } \\
\hline & 1 & 2 & 3 & 4 & 5 & 6 & 7 & 8 & 9 & 10 & 11 \\
\hline \multicolumn{12}{|l|}{ Funcionalidade } \\
\hline 1. Cognição & & $0,65^{*}$ & $0,74^{*}$ & $0,61^{*}$ & $0,80^{*}$ & $0,78^{*}$ & $0,85^{*}$ & $-0,11$ & $-0,10$ & $0,56^{*}$ & 0,17 \\
\hline 2. Mobilidade & & & $0,62^{*}$ & $0,51^{*}$ & $0,76^{*}$ & $0,77^{*}$ & $0,87^{*}$ & $-0,33^{*}$ & $-0,40^{*}$ & $0,50 *$ & $0,40^{*}$ \\
\hline 3. Autocuidado & & & & $0,61^{*}$ & $0,73^{*}$ & $0,74^{*}$ & $0,74^{*}$ & 0,03 & $-0,13$ & $0,58^{*}$ & 0,15 \\
\hline 4. Relações interpessoais & & & & & $0,55^{*}$ & $0,54^{*}$ & $0,67^{*}$ & $-0,05$ & 0,01 & $0,42^{*}$ & $-0,01$ \\
\hline 5. AVD & & & & & & $0,89^{*}$ & $0,85^{*}$ & $-0,17$ & $-0,32^{*}$ & $0,59 *$ & $0,33^{*}$ \\
\hline 6. Participação social & & & & & & & $0,85^{*}$ & $-0,22^{*}$ & $-0,30^{*}$ & $0,51^{*}$ & $0,35^{*}$ \\
\hline 7. Escore total & & & & & & & & $-0,25^{*}$ & $-0,23^{*}$ & $0,60 *$ & $0,31^{*}$ \\
\hline 8. FM MMSS & & & & & & & & & $0,45^{\star}$ & $-0,11$ & $-0,59$ * \\
\hline 9. FM MMII & & & & & & & & & & $-0,17$ & $-0,62^{*}$ \\
\hline 10. Medo de cair & & & & & & & & & & & 0,19 \\
\hline 11. Risco de queda & & & & & & & & & & & \\
\hline
\end{tabular}

Correlação significante:* ${ }^{2}<0,05$; Correlação de Pearson; AVD: atividades de vida diária; FM: força muscular; MMSS: membros superiores; MMII: membros inferiores

Tabela IV - Análise de regressão múltipla usando os domínios de funcionalidade e as variáveis de força muscular como preditores do medo de cair dos idosos. Maringá, Paraná, Brasil, 2019.

\begin{tabular}{|c|c|c|c|c|c|}
\hline Variáveis & B padronizado & $\mathbf{R}^{2}$ ajustado & $\mathbf{p}$ & VIF & DW \\
\hline Cognição & 0,072 & \multirow{8}{*}{0,40} & 0,702 & 4,295 & \multirow{8}{*}{1,81} \\
\hline Mobilidade & $-0,139$ & & 0,392 & 3,155 & \\
\hline Autocuidado & 0,409 & & $0,005^{\star}$ & 2,645 & \\
\hline Relações interpessoais & 0,034 & & 0,794 & 2,389 & \\
\hline Atividades da vida diária & 0,379 & & $0,047^{*}$ & 4,495 & \\
\hline Participação social & $-0,053$ & & 0,785 & 4,491 & \\
\hline FM MMSS & 0,025 & & 0,798 & 1,824 & \\
\hline FM MMII & 0,112 & & 0,309 & 2,164 & \\
\hline
\end{tabular}

Associação significante: * $\mathrm{p}<0,05$; Análise de regressão múltipla; VIF: Fatores de Inflação de Variância; DW: Durbin-Watson; FM: força muscular; MMSS: membros superiores; MMII: membros inferiores

Tabela V - Análise de regressão múltipla usando os domínios de funcionalidade e as variáveis de força muscular como preditores do risco de queda dos idosos. Maringá, Paraná, Brasil, 2019.

\begin{tabular}{|c|c|c|c|c|c|}
\hline Variáveis & B padronizado & $\mathbf{R}^{2}$ ajustado & $\mathbf{p}$ & VIF & DW \\
\hline Cognição & 0,082 & \multirow{8}{*}{0,51} & 0,626 & 4,384 & \multirow{8}{*}{1,86} \\
\hline Mobilidade & 0,075 & & 0,606 & 3,396 & \\
\hline Autocuidado & 0,161 & & 0,208 & 2,588 & \\
\hline Relações interpessoais & $-0,340$ & & $0,004^{*}$ & 2,156 & \\
\hline Atividades da vida diária & 0,239 & & 0,195 & 4,434 & \\
\hline Participação social & $-0,077$ & & 0,662 & 4,727 & \\
\hline Força muscular de MMSS & $-0,512$ & & $0,001^{*}$ & 1,239 & \\
\hline Força muscular de MMII & $-0,192$ & & $0,048^{*}$ & 1,576 & \\
\hline
\end{tabular}

Associação significante: * $p<0,05$; Análise de regressão múltipla; VIF: Fatores de Inflação de Variância; DW: Durbin-Watson; MMSS: membros superiores; MMII: membros inferiores

\section{DISCUSSÃO}

Os principais resultados do estudo apontaram que a piora na funcionalidade, no autocuidado e nas AVD prediz o medo de queda em idosos. Quanto maior a força muscular, menor o risco de queda dos idosos e quanto pior a funcionalidade nas relações interpessoais, menor é o risco de queda. 
Em relação ao impacto da força muscular sobre o risco de queda dos idosos, estudiosos ${ }^{(21)}$ sugerem que a partir da sexta década de vida a redução da força muscular em MMSS e MMII torna-se mais evidente, e que essa redução é ainda mais expressiva nos MMII. Outro estudo concluiu que a baixa força muscular associou-se à ocorrência de quedas no último ano em relação aos idosos avaliados. Em idosos, o déficit no equilíbrio postural pode dificultar a recuperação rápida diante de pequenas perturbações posturais, tornando o indivíduo mais suscetível a quedas ${ }^{(22)}$. A força de MMII está intimamente relacionada à capacidade de equilíbrio( ${ }^{(23)}$, que, por sua vez, leva o indivíduo a evitar quedas.

Ainda sobre esse dado, um estudo, ao comparar o equilíbrio e força máxima de MMIl de idosos praticantes de musculação, praticantes de hidroginástica e sedentários, verificou que a força máxima de MMII era diretamente proporcional ao equilíbrio funcional e dinâmico ${ }^{(24)}$. Outros pesquisadores encontraram que idosos não caidores apresentaram melhor nível de força de MMII em relação aos idosos caidores ${ }^{(25)}$. Além disso, uma revisão de literatura verificou que a força muscular de MMII reduz a ocorrência de queda em idosos, assim como idosos com melhor nível de força muscular também apresentam equilíbrio melhor, o que é um fator que também colabora para a redução das quedas ${ }^{(26)}$.

Também foi evidenciado no atual estudo que, quanto pior a funcionalidade de relações interpessoais, menor é o risco de quedas nos idosos. Esse é um dado controverso, visto que idosos caidores têm tendência ao desenvolvimento do isolamento social e depressão, de modo que a reclusão e a restrição ao quarto, por exemplo, acabam sendo uma saída para evitar novas quedas e, com isso, o ciclo de redução das relações interpessoais acaba se perpetuando. Salienta-se que a ocorrência de queda tem desdobramentos complexos ${ }^{(6)}$. Esses desdobramentos atingem os domínios físico, emocional, psicossocial e familiar, além de poder acarretar a depressão, hospitalização e até a morte ${ }^{(6)}$.

No presente estudo, a piora da funcionalidade nas AVD e, por conseguinte, do autocuidado foi capaz de predizer a piora no medo de cair em idosos. As AVD referem-se às tarefas necessárias para o cuidado com corpo ou autopreservação, autocuidado. O autocuidado é um conceito importante dentro da saúde do idoso. Compreende-se que idosos capazes de realizar o seu autocuidado com eficiência apresentam um bom aspecto geral de saúde e até mesmo a preservação de sua cognição(27).

Realizar AVD sugere um estado considerável da preservação da força muscular e do equilíbrio, portanto pode-se inferir que evitar realizar AVD ou mesmo não conseguir realizá-la sugere que o idoso esteja tentando se preservar de uma possível queda ou que suas habilidades motoras estejam apresentando déficits que o impossibilitem de realizar algumas tarefas cotidianas. Vale ressaltar que a AVD é usada para medir a capacidade funcional. No presente estudo, além de estar associada ao medo de cair, ainda sugere um impacto negativo nas funções, estrutura do corpo e cognição(21).

Sob a perspectiva da promoção da saúde, que perpassa a atenção primária e abrange também políticas públicas e questões técnicas em torno do processo saúde-doença-cuidado, é possível afirmar que a queda é o desfecho negativo de uma cascata de efeitos deletérios do organismo ${ }^{(28)}$. $\mathrm{O}$ impacto econômico relacionado à queda evidencia custos elevados $^{(28,29)}$. Evitar essa deleção e, por conseguinte, a queda demandará a articulação de recursos técnicos, pessoais, políticos e comunitários ${ }^{(28)}$.

Diante disso, reforça-se que a queda é um problema de saúde pública com impactos expressivos sobre a funcionalidade e a saúde física e mental do idoso, podendo afetar também suas relações interpessoais e o meio no qual está inserido.

Algumas limitações do presente estudo devem ser consideradas, entre elas: estudo de delineamento transversal, que não possibilita explorar as relações de causa e efeito entre as variáveis de interesse; algumas medidas de avaliação foram coletadas por meio de questionários e não da mensuração objetiva, o que pode afetar parcialmente a confiabilidade dos dados coletados; e não foi avaliada a presença de morbidades dos idosos, que pode impactar nos resultados de funcionalidade, força e queda.

\section{CONCLUSÃO}

Observa-se que as medidas de funcionalidade e a força muscular estão associadas ao risco de queda e ao medo de cair na população de idosos em questão.

\section{CONFLITOS DE INTERESSE}

Os autores declaram a inexistência de conflitos de interesses, tanto na execução das ações do projeto de pesquisa como na escrita deste manuscrito. 


\section{CONTRIBUIÇÕES}

Daniel Vicentini de Oliveira, Felipe Carmona Yamashita, José Roberto Andrade do Nascimento Júnior e Sônia Maria Marques Gomes Bertolini contribuíram com a elaboração e o delineamento do estudo; a aquisição, análise e interpretação de dados; e a redação e/ou revisão do manuscrito. Naelly Renata Saraiva Pivetta e Matheus Amarante do Nascimento contribuíram com a redação e/ou revisão do manuscrito. Natália Quevedo dos Santos contribuiu com a aquisição, análise e interpretação de dados.

\section{REFERÊNCIAS}

1. Mendonça TV, Rego AS, Mendonça FMA, Silva, Vieira JNL. Efeitos do uso de um programa cinesioterapêutico na força de preensão manual de idosas. Rev Investig Bioméd [Internet]. 2018 [acesso em 2020 Jul 15];10(1):56-8. doi: 10.24863/rib.v10i1.175

2. Thompson WRDLS, Pescatello RA. Diretrizes do ACSM-para os testes de esforço e sua prescrição. Rio de Janeiro: Ganabara Koogan; 2010.

3 Ribeiro DKDMN, Lenardt MH, Lourenço TM, Betiolli SE, Seima MD, Guimarães CA. The use of the functional independence measure in elderly. Rev Gaucha Enferm. 2018;38(4).

4. Fernandes KC, Oliveira RC, Freitas AC, Souza AA, Lopes RA. Associação entre função física e incapacidade autorrelatada em idosos comunitários: uma abordagem de acordo com o Modelo de Funcionalidade, Incapacidade e Saúde. ConScientiae Saúde [Internet]. 2019 [acesso em 2020 Jul 15];18(2):209-17. doi: 10.5585/conssaude.v18n2.11142

5. Bizovska L, Svoboda Z, Janura M, Bisi MC, Vuillerme N. Local dynamic stability during gait for predicting falls in elderly people: a one-year prospective study. PLoS One [Internet]. 2018 [acesso em $2020 \mathrm{Jul}$ 15];13(5):0197091. doi: 10.1371/journal.pone.0197091

6. Moraes SA, Soares WJS, Lustosa LP, Bilton TL, Ferrioli E, Perracini MR. Characteristics of falls in elderly persons residing in the community: a population-based study. Rev Bras Geriatr Gerontol [Internet]. 2017 [acesso em 2020 Jul 15];20(5):691-701. doi: 10.1590/1981-22562017020.170080

7. Silva IGP, Peruzzo HE, Lino IGT, Marquete VF, Marcon SS. Perfil sociodemográfico e clínico de idosos em risco de quedas no sul do Brasil. J Nurs Heal [Internet]. 2019 [acesso em 2020 Jul 15];9(3):1-12. doi: 10.15210/jonah.v9i3.16808

8. Oliveira T, Baixinho CL, Henriques MA. Risco multidimensional de queda em idosos. Rev Bras Promoç Saúde [Internet]. 2018 [acesso em 2020 Jul 15];31(2):1-9. doi: 10.5020/18061230.2018.7058

9. World Health Organization. Global recommendations on physical activity for health [Internet]. 2010 [acesso em 2020 Jul 15]. Disponível em: https://apps.who.int/iris/bitstream/handle/10665/44399/9789241599979_ eng.pdf;jsessionid=6EFD1359778000FB8E42EA85A4C8DB32? sequence $=1$

10. Soares WJS, Moraes SA, Ferriolli E, Perracini MR. Fatores associados a quedas e quedas recorrentes em idosos: estudo de base populacional. Rev Bras Geriatr Gerontol [Internet]. 2014 [acesso em 2020 Jul 15];17(1):49-60. doi: 10.1590/S1809-98232014000100006

11. Santos RKM, Maciel ÁCC, Britto HMJS, Lima JCC, Souza TO. Prevalência e fatores associados ao risco de quedas em idosos adscritos a uma Unidade Básica de Saúde do município de Natal, RN, Brasil. Cienc Saude Colet [Internet]. 2015 [acesso em 2020 Jul 15];20(12):3753-62. doi: 10.1590/1413-812320152012.00662015

12. Morsch $P$, Myskiw M, Myskiw JC. A problematização da queda e a identificação dos fatores de risco na narrativa de idosos. Cienc Saude Colet [Internet]. 2016 [acesso em 2020 Jul 15];21(11):3565-74. doi: 10.1590/1413-812320152111.06782016

13. Rezende CP, Gaede-Carrillo MRG, Sebastião ECO. Queda entre idosos no Brasil e sua relação com o uso de medicamentos: revisão sistemática. Cad Saude Publica [Internet]. 2012 [acesso em 2020 Jul 15];28(12):2223-35. doi: 10.1590/S0102-311X2012001400002

14. Santos SCA, Figueiredo DMP. Preditores do medo de cair em idosos portugueses na comunidade: um estudo exploratório. Cienc Saude Colet [Internet]. 2019 [acesso em 2020 Jul 15];24(1):77-86. doi: 10.1590/141381232018241.29932016 
15. Pinheiro PA, Passos TD-RO, Coqueiro RS, Fernandes MH, Barbosa AR. Desempenho motor de idosos do Nordeste brasileiro: diferenças entre idade e sexo. Rev Esc Enferm USP [Internet]. 2013 [acesso em 2020 Jul 15];47(1):128-36. doi: 10.1590/S0080-62342013000100016

16. Brucki SMD, Nitrini R, Caramelli P, Bertolucci PHF, Okamoto IH. Sugestões para o uso do mini-exame do estado mental no Brasil. Arq Neuropsiquiatr [Internet]. 2003 [acesso em 2020 Jul 15];61(3B):777-81. doi: 10.1590/S0004-282X2003000500014

17. Camargos FFO, Dias RC, Dias JMD, Freire MTF. Adaptação transcultural e avaliação das propriedades psicométricas da Falls Efficacy Scale - International em idosos brasileiros (FES-I-BRASIL). Rev Bras Fisioter [Internet]. 2010 [acesso em 2020 Jul 15];14(3):237-43. doi: 10.1590/S1413-35552010000300010

18. Silva C, Coleta I, Silva AG, Amaro A, Alvarelhao J, Queiros A, et al. Adaptação e validacao do WHODAS 2.0 em utentes com dor musculoesqueletica. Rev Saude Pública [Internet]. 2013 [acesso em $2020 \mathrm{Jul}$ 15];47(4):752-8. doi: 10.1590/S0034-8910.2013047004374

19. Rikli RE, Jones CJ. Teste de Aptidão Física para Idosos. São Paulo: Manole; 2008.

20. Tabachnick B, Fidell L. Using Multivariate Statistics. [Local desconhecido]: Allynand; 2001.

21. Borges V, Silva N, Malta AC, Xavier NC, Santana Bernardes LE. Falls, Muscle Strength, and Functional Abilities in Community-Dwelling Elderly Women. Arch Phys Med Rehabil [Internet]. 2017 [acesso em 2020 Jul 15];98(10):e22. doi: 10.1590/1980-5918.030.002.ao16

22. Howcroft J, Lemaire ED, Kofman J, Mcllroy WE. Elderly fall risk prediction using static posturography. PLoS One [Internet]. 2017 [acesso em 2020 Jul 15]21;12(2):e0172398. doi: 10.1371/journal.pone.0172398

23. Costa LSV, Sousa NM, Alves AG, Alves FAVB, Araújo RF, Nogueira MS. Análise comparativa da qualidade de vida, equilíbrio e força muscular em idosos praticantes de exercício físico e sedentários. Rev Fac Montes Belos [Internet]. 2015 [acesso em 2020 Jul 15];8(3):161-79. Disponível em: http:// http://revista.fmb.edu.br/ index. php/fmb/article/view/190/179

24. Souza L, Coelho B, Freire B, Delevatti R, Roncada C, Tiggemann C, et al. Comparação dos níveis de força e equilíbrio entre idosos praticantes de musculação e de hidroginástica. Rev Bras Ativ Fís Saúde [Internet]. 2014 [acesso em 2020 Jul 15];19(5). doi: 10.12820/rbafs.v.19n5p647

25. Gonçalves AK, Griebler EM, Possamai VD, Costa RR, Martins VF. Idosos caidores e não caidores: programa de exercício multicomponente e prevalência de quedas. ConScientiae Saúde [Internet]. 2017 [acesso em 2020 Jul 15];16(2):187-93. doi: 10.5585/conssaude.v16n2.6987

26. Castro Finamore ALM. Efeito dos exercícios multimodais na prevenção de quedas em idosos ativos revisão de literatura [trabalho de conclusão de curso] [Internet]. Belo Horizonte: UFMG; 2019 [acesso em 2020 Jul 15]. Disponível em: https://repositorio.ufmg.br/handle/1843/30520

27. Pivetta NRS, Marincolo JCS, Neri AL, Aprahamian I, Yassuda MS, Borim FSA. Multimorbidity, frailty and functional disability in octogenarians: A structural equation analysis of relationship. Arch Gerontol Geriatr [Internet]. 2020 [acesso em 2020 Jul 15];86:103931. doi: 10.1016/j.archger.2019.103931

28. Crizzle AM, Dykeman C, Laberge S, MacLeod A, Olsen-Lynch E, Brunet F, et al. A public health approach to mobilizing community partners for injury prevention: a scoping review. PLoS one. 2019;14(1).

29. Grysztar M, Duplaga M, Wojcik S, Rodzinka M. Eficácia de intervenções de promoção da saúde lideradas por pares dirigidas a idosos: revisão sistemática Marcin Grysztar. Eur J Public Health. 2017;27(suppl_3).

\section{Endereço para correspondência:}

Daniel Vicentini de Oliveira

Centro Universitário de Maringá

Avenida Guedner, 1610

Bairro: Jardim Aclimação

CEP: 87050-900 - Maringá - PR - Brasil

E-mail: d.vicentini@hotmail.com 